DOI: 10.4274/jarem.galenos.2020.3255

J Acad Res Med 2020;10(2):204-7

\title{
Is Mucopolysaccharidosis a Cause of Sleep and Speech Disorders? Report of Four Cases?
}

\author{
(1) Nafiye Urgancl1, (1) Derya Kalyoncu22, (1) Reyhan Gümüştekin2 \\ ${ }^{1}$ University of Health Sciences Turkey, Şişli Etfal Training and Research Hospital, Clinic of Pediatric Gastroenterology, İstanbul, Turkey \\ 2University of Health Sciences Turkey, Şişli Etfal Training and Research Hospital, Clinic of Pediatrics, İstanbul, Turkey
}

Cite this article as: Urgancı N, Kalyoncu D, Gümüştekin R. Is Mucopolysaccharidosis a Cause of Sleep and Speech Disorders? Report of Four Cases? J Acad Res Med 2020;10(2):204-7

\begin{abstract}
Mucopolysaccharidosis type III (MPS 3) is an autosomal recessive disorder caused by a deficiency of one of the four enzymes involved in the lysosomal degradation of heparan sulfate (HS). It is often unrecognized or misdiagnosed in children as an idiopathic developmental/speech delay, attention deficit/hyperactivity disorder (ADHD), and/or autism spectrum disorder. It is characterized by progressive mental deterioration and behavioral problems with dysmorphic facial features and mild somatic signs. We report on children with ADHD who were repeatedly admitted to the pediatric psychiatry department for sleep disturbances, hyperactivity, and speech delay; and to the emergency department with accidental corrosive substance ingestion. Children with mental retardation, coarse face, and hypertrichosis were referred to the pediatric gastroenterology department for preliminary diagnosis of MPS. Lysosomal enzyme activity examinations in leukocytes revealed increased levels of total glycosaminoglycans, heparin, and HS, and decreased HS sulphamidase activity, leading to the diagnosis of MPS III.

Keywords: Children; mucopolysaccharidosis, sanfilippo, attention deficit/hyperactivity disorder
\end{abstract}

\section{Introduction}

Mucopolysaccharidoses (MPSs) are a group of seven inherited metabolic disorders that are characterized by the absence or deficiency of specific lysosomal enzymes that lead to the accumulation of glycosaminoglycans (GAGs) within the lysosomes. MPS type III (or Sanfilippo syndrome) is the most common type and is composed of four different subtypes: type A (OMIM \#252900), type B (OMIM \#252920), type C (OMIM \#252930), and type D (OMIM \#252940). Each subtype is caused by a deficiency of a different enzyme in the catabolic pathway of heparan sulfate (HS), a type of GAG. MPSs comprise 11 lysosomal diseases, each with an absence of a specific step in the degradation of GAGs that leads to their accumulation in tissues and to a range of clinical consequences, including central nervous system (CNS) impairment, depending on the type of the $\operatorname{MPS}(1,2)$

Seven types of MPS disorders caused by deficiencies of 10 different enzymes are as follows: MPS I (Hurler MIM 607014, Hurler-Scheie MIM 607015, Scheie MIM 607016), MPS II (Hunter syndrome MIM 309900), MPS III A (MIM 252900), B (OMIM 252920), C (MIM 252930), or D (MIM 252940) (Sanfilippo syndrome), MPS IV A (MIM 253000) or B (MIM 253010) (Morquio syndrome), MPS VI (Maroteaux-Lamy syndrome, MIM 253200), MPS VII (Sly disease, MIM 253220), and MPS IX (MIM 601492) (1). The clinical symptoms vary widely across the different subtypes (1).

MPSs are a group of inherited metabolic disorders caused by a deficiency of lysosomal enzymes that take part in the

ORCID IDs of the authors: N.U. 0000-0003-4854-507X; D.K., 0000-0001-8449-7621; R.G. 0000-0010-6544-2344. 
She had severe behavioral disorder, hyperactivity, mental retardation, speech disorder, coarse face and hypertrichosis. Her laboratory investigations revealed normal complete blood count, liver function, serum copper, ceruloplasmin, alpha 1-antitrypsin levels, and thyroid function. Hepatitis $A, B$, and $C$ were ruled out on serology. Urinary GAGS were significantly increased. The lysosomal enzyme activity was assessed in leukocytes and showed that total GAG levels were $63.5 \mathrm{mg} / \mathrm{mmol}$; levels of creatinin, heparin, and HS were increased; whereas, HS sulphamidase activity was decreased $(0 \mathrm{nmol} / \mathrm{mg}$ protein $/ 17 \mathrm{~h})$ compared with the normal reference range $(3.2 \pm 20.4 \mathrm{nmol} / \mathrm{mg}$ protein $/ 17 \mathrm{~h})$. She was thus diagnosed with MPS III A.

Chest radiography revealed beaking of vertebral bodies and oar shaped ribs (Figure 1). Abdominal USG showed hepatosplenomegaly. Echocardiography and ophthalmic examinations were normal. Severe sensorineural hearing loss was detected.

\section{Case 2}

An 11-year-old boy was admitted to the pediatric emergency department after ingesting a corrosive substance.

Upper gastrointestinal tract endoscopy revealed mild hyperemia. $\mathrm{He}$ had severe behavioral disorder, hyperactivity, mental retardation, speech disorder, coarse face, and hypertrichosis, similar to his sibling.

He had previously been admitted to the pediatric psychiatry department several times for walk and speech delay. He had severe mental retardation, hyperactivity, speech disorder, and sleep disturbances since he was 6 years old and was diagnosed with ADHD. His physical examination was unremarkable except for the presence of a coarse face and hypertrichosis. There was no organomegaly.

His laboratory investigations revealed normal complete blood count, liver function, serum copper, ceruloplasmin, alpha 1-antitrypsin levels, and thyroid function. Hepatitis A, B, and C were ruled out. Urinary GAGs were increased. Total GAG levels

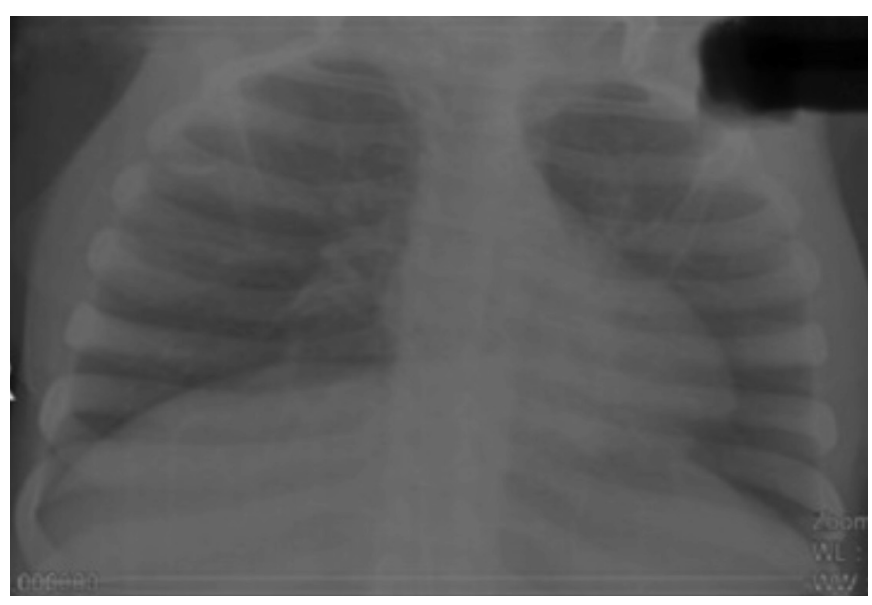

Figure 1. Chest radiograph showing beaking of vertebral bodies and oar shape ribs 
were $41.8 \mathrm{mg} / \mathrm{mmol}$; levels of creatinin, heparin, and HS were increased; whereas, HS sulphamidase activity was decreased (0.1 nmol/mg protein/17 h). He was diagnosed with MPS III A. Chest radiograph revealed beaking of vertebral bodies and oar shaped ribs. Sensorineural hearing loss was observed; while, echocardiography and ophthalmic examinations were normal.

\section{Case 3}

A 4.5-year-old boy was admitted to the pediatric emergency department after ingesting a corrosive substance (bleach). Upper gastrointestinal tract endoscopy revealed no pathology.

His history revealed no consanguinity. He had agitation, hyperactivity, and short sleep duration. He had started to talk and walk after 2.5 years, and had been followed-up by pediatric psychiatry department for the last 6 months with the diagnosis ADHD.

His physical examination revealed a coarse face, roughness of the hands and feet. There was no organomegaly. The lysosomal enzyme activity was approximately $0 \mathrm{nmol} / \mathrm{mg}$ protein $/ 17 \mathrm{~h}$ that led to the diagnosis of MPS III A. He had no skeletal abnormalities or hearing loss.

\section{Case 4}

A 14-year-old boy was admitted to the pediatric emergency department with complaints of corrosive substance ingestion. Upper gastrointestinal tract endoscopy revealed linear erosions and hyperemia. His oral intake was stopped and proton-pump inhibitors were administered.

His history revealed no consanguinity. He had started to talk at 4.5 years, and suffered from sleep disorders since the age of 8 years. $\mathrm{He}$ had been admitted to the pediatric psychiatry department many times with the suspicion of ADHD.

His physical examination revealed a coarse face, hypertrichosis, and roughness of the hands and feet. He had hearing loss and skeletal abnormalities. Liver was palpable $3 \mathrm{~cm}$ below right costal margin. His laboratory investigations were normal. As the lysosomal enzyme activity was $0 \mathrm{nmol} / \mathrm{mg}$ protein $/ 17 \mathrm{~h}$, a diagnosis of MPS III A was made. A repeat endoscopy performed 1 week later was unremarkable.

Written informed consents were obtained from all the patients and/or their parents.

\section{DISCUSSION}

MPS III A is an autosomal recessive disorder caused by a deficiency of heparan $\mathrm{N}$-sulphatase that is involved in the lysosomal degradation of HS. It is reportedly the most severe form, with an earlier onset and faster progression of symptoms than in the other types of MPSs $(3-6,8)$. MPS III A is characterized by progressive mental retardation, speech delay, sleep disturbances, and behavioral disorders (3-11). It has been reported that $40.6 \%$ of the patients talked after the $15^{\text {th }}$ month of life, $7.2 \%$ of them walked after the $18^{\text {th }}$ month, and $26.1 \%$ had both delayed talking and walking (5). The patients were usually diagnosed at $3-4.5$ years of age $(5,10)$.

The first clinical symptoms reported by Meyer et al. (5) included behavioral and sleep disturbances; whereas, speech (48\%) and behavioral disorders (9\%) were cited as the first symptoms by Buhrman et al. (10). Our patients were admitted to the pediatric psychiatry department for sleep disorders, hyperactivity, and delayed speech and walking. No clinical improvement occurred during their follow-up period. The diagnosis of MPS was based on the physical signs, such as a coarse face (flat nasal bridge, thickened lips, low set ears, macroglossia, short neck, hypertrichosis, etc.), hepatosplenomegaly, broad hands, and short fingers.

Although, skeletal abnormalities are less prominent in MPS III than in the other types of MPS, regular imaging of the spine, hips, and the lower extremities is recommended in these patients (12). Three of our patients had skeletal abnormalities.

Leukocytes and/or skin fibroblast cultures are the gold standard for establishing the diagnosis and determining the subtype of MPS. Prenatal diagnosis is possible, and familial carriers can be identified by molecular genetic testing. There is no specific enzyme replacement therapy; however, hematopoietic stem cell transplantation can be used (13).

In conclusion, MPSs can be easily misdiagnosed as other common diseases seen in children, and this can result in their inappropriate management. Therefore, pediatricians, pediatric psychiatrists, and physicians must suspect MPSs in patients who present with hyperactivity, speech delay or deterioration, autism-like behavioral disorders, and motor developmental delay, even in the absence of apparent features like coarse face.

Informed Consent: Written informed consents were obtained from all the patients and/or their parents.

Peer-review: Externally and internally peer-reviewed.

Author Contributions: Surgical and Medical Practices - N.U.; Concept N.U., D.K.; Design - N.U., D.K., R.G.; Data Collection and/or Processing - N.U., R.G.; Analysis and/or Interpretation - N.U., D.K.; Literature Search - D.K.; Writing Manuscript - D.K.

Conflict of Interest: The authors have no conflict of interest to declare.

Financial Disclosure: The authors declared that this study has received no financial support.

\section{REFERENCES}

1. Neufeld E., Muenzer J. The Mucopolysaccharidoses. The Online Metabolic and Molecular Bases of Inherited Disease. OMMBID McGrawHill Medical. 8th ed. McGraw-Hill; New York, NY, USA: 2001.

2. Poswar F.D.O, Vairo F, Burin M, Michelin-Tirelli K, Brusius-Facchin A.C, Kubaski F, Souza C.F.M.D, Baldo G, Giugliani R. Lysosomal diseases: Overview on current diagnosis and treatment. Genet. Mol. Biol. 2019;42:165-177.

3. Valstar MJ, Ruijter GJG, van Diggelen OP, Poorthuis BJ, Wijburg FA. Sanfilippo syndrome: a mini-review. J Inherit Metab Dis 2008; 31: 240-52.

4. Wijburg FA, Węgrzyn G, Burton BK, Tylki-Szymańska A. Mucopolysaccharidosis type III (Sanfilippo syndrome) and misdiagnosis of idiopathic developmental delay, attention deficit/hyperactivity disorder or autism spectrum disorder. Acta Paediatr 2013; 102: 462-70.

5. Meyer A, Kossow K, Gal A, Mühlhausen C, Ullrich K, Braulke T, et al. 
Scoring evaluation of the natural course of mucopolysaccharidosis type IIIA (Sanfilippo syndrome type A). Pediatrics 2017; 120: 1255-61.

6. Delgadillo V, O'Callaghan Mdel M, Gort L, Coll MJ, Pineda M. Natural history of Sanfilippo syndrome in Spain. Orphanet J Rare Dis 2013; 8: 189.

7. Valstar MJ, Marchal JP, Grootenhuis M, Colland V, Wijburg FA. Cognitive development in patients with mucopolysaccharidosis type III (Sanfilippo syndrome). Orphanet J Rare Dis 2011; 6: 43.

8. Ruijter GJ, Valstar MJ, van de Kamp JM, van der Helm RM, Durand S, van Diggelen $O P$, et al. Clinical and genetic spectrum of Sanfilippo type C (MPS IIIC) disease in The Netherlands. Mol Genet Metab 2008; 93: 10411

9. Clarke LA, Winchester B, Giugliani R, Tylki-Szymańska A, Amartino H. Biomarkers for the mucopolysaccharidoses: Discovery and clinical utility. Mol Genet Metab 2012; 106: 395-402.
10. Buhrman D, Thakkar K, Poe M, Escolar ML. Natural history of Sanfilippo syndrome type A. J Inherit Metab Dis 2014; 37: 431-7.

11. Cross EM, Hare DJ. Behavioural phenotypes of the mucopolysaccharide disorders: a systematic literature review of cognitive, motor, social, linguistic and behavioural presentation in the MPS disorders. J Inherit Metab Dis 2013; 36: 189-200.

12. White KK. Orthopaedic aspects of mucopolysaccharidoses. Rheumatology (Oxford) 2011; 50(Suppl 5): 26-33.

13. Burrow TA, Hopkin RJ, Leslie ND, Tinkle BT, Grabowski GA. Enzyme reconstitution/replacement therapy for lysosomal storage diseases. Curr Opin Pediatr 2017; 19: 628-35. 